\title{
Invarianza Factorial Según Sexo de la Basic Empathy Scale Abreviada en Adolescentes Peruanos
}

\section{Factor Invariance Across Sex of the Abbreviated Basic Empathy Scale in Peruvian Adolescents}

\author{
José Ventura-León y Tomás Caycho-Rodríguez \\ Universidad Privada del Norte \\ Sergio Dominguez-Lara \\ Universidad de San Martín de Porres
}

\begin{abstract}
Si bien la Basic Empathy Scale abreviada (BES abreviada) ha sido utilizada para estudiar las diferencias de la empatía entre hombres y mujeres adolescentes, no se sabe si el constructo es medido de forma similar en varones y mujeres, es decir, si existe invarianza factorial en función del sexo. El estudio tuvo como objetivo analizar la invarianza factorial de la BES abreviada en función del sexo en una muestra por conveniencia de estudiantes secundarios (480 varones y 454 mujeres) de 3 instituciones educativas de Lima Metropolitana, Perú. Un análisis factorial confirmatorio multigrupo mostró que el modelo de 2 factores (empatía cognitiva y afectiva) es factible para ambos sexos. La invarianza configural verifica la estructura bidimensional, llegando a un cumplimiento satisfactorio de invarianza fuerte. La prueba $t$ de Student reveló que las mujeres presentan mayor empatía cognitiva y afectiva que los varones. Se concluye que existe evidencia empírica que respalda la invarianza factorial parcial de la BES abreviada en adolescentes peruanos según el sexo. Estudios futuros deben replicar estos procedimientos en participantes con diferentes características culturales y otros rangos de edad.
\end{abstract}

Palabras clave: Basic Empathy Scale, empatía, invarianza factorial, adolescentes, sexo

\begin{abstract}
Although the abbreviated Basic Empathy Scale (abbreviated BES) has been used to study the differences of empathy between adolescent men and women, it is unknown whether the construct is measured similarly in both, that is, whether there is factor invariance across sex. The aim of the study was to analyze the factor invariance of the abbreviated BES across sex in a convenience sample of high-school students (480 males and 454 females) attending 3 schools in Lima Metropolitana, Peru. A multigroup confirmatory factor analysis showed that the 2-factor model (cognitive and affective empathy) is feasible for both sexes. Configural invariance verifies the two-dimensional structure, reaching a satisfactory compliance of strong invariance. Student's $t$-test revealed that women have greater cognitive and affective empathy than men. It is concluded that there is empirical evidence to support the partial factor invariance across sex of the abbreviated BES in Peruvian adolescents. Future studies should replicate these procedures in participants with different cultural characteristics and other age ranges.
\end{abstract}

Keywords: Basic Empathy Scale, adolescents, empathy, factor invariance, sex

La empatía es una variable importante para el desarrollo y buen funcionamiento de las relaciones interpersonales (Guzmán González, Péloquin, Lafontaine, Trabucco \& Urzúa, 2014). Si bien, en términos generales, la empatía se conceptualiza como la capacidad para comprender y experimentar los sentimientos de otros (Borrell i Carrió, 2004; Campbell \& Babrow, 2004; Jolliffe \& Farrington, 2006a), su estudio científico es de difícil abordaje debido, en buena parte, a la complejidad y multidimensionalidad del constructo (Fernández-Pinto, López-Pérez \& Márquez, 2008; Rey, 2003). Esto se ve reflejado en la existencia de diversas definiciones que se expresan en las más de 40 medidas de empatía que existen (Hemmerdinger, Stoddart \& Lilford, 2007; Pedersen, 2008). Estas medidas revelan las diferentes concepciones de la empatía desde una perspectiva cognitiva, afectiva o ambas.

Existe un consenso general que sugiere que la empatía se compone de dos dimensiones, la empatía afectiva y la cognitiva (Jolliffe \& Farrington 2004; Shamay-Tsoory, 2011). Si bien algunos autores sugieren la presencia de una tercera dimensión motora, caracterizada por expresiones faciales, voces, gestos y posturas

José Ventura-León y Tomás Caycho-Rodríguez, Carrera de Psicología, Facultad de Ciencias de la Salud, Universidad Privada del Norte, Lima, Perú; Sergio Dominguez-Lara, Instituto de Investigación de Psicología, Escuela Profesional de Psicología, Facultad de Ciencias de la Comunicación, Turismo y Psicología, Universidad de San Martín de Porres, Lima, Perú.

La correspondencia relativa a este artículo debe ser dirigida a José Ventura-León, Carrera de Psicología, Facultad de Ciencias de la Salud, Universidad Privada del Norte, Av. Tingo María 1122, Lima, Perú. E-mail: jose.ventura@upn.pe 
de los demás, inducidas por experiencias emocionales vicarias (Dimberg \& Thunberg, 2012; Dimberg, Thunberg \& Elmehed, 2000), el presente estudio se centrará solo en las dos primeras dimensiones.

La dimensión cognitiva de la empatía se conceptualiza típicamente como una habilidad mental expresada en una serie de procesos de toma de perspectiva que utiliza una persona para imaginar la situación del otro, con el fin de identificar y comprender sus sentimientos (Decety, 2010; Jolliffe \& Murray, 2012; Neumann et al., 2012; Sulzer, Feinstein \& Wendland, 2016). La dimensión afectiva se caracteriza por la susceptibilidad para experimentar y compartir los sentimientos de otra persona (Eisenberg, Shea, Carlo \& Knight, 1991; Jolliffe \& Murray, 2012). Desde una perspectiva neurocientífica, si bien la empatía cognitiva y afectiva son complementarias, se encuentran diferenciadas desde un punto de vista funcional y neurobiológico (Arán Filippetti, López \& Richaud, 2012; Shamay-Tsoory, Aharon-Peretz \& Perry, 2009; Shirtcliff, Dahl \& Pollak, 2009; van Boxtel, 2010). En este sentido, la empatía afectiva está asociada al sistema de neuronas espejo, que constituye un sistema básico de contagio afectivo y emocional, mientras que la empatía cognitiva se relaciona con procesos de toma de perspectiva asociados con las funciones cognitivas de alto nivel (Arán Filippetti et al., 2012; Decety \& Jackson, 2004; Shamay-Tsoory et al., 2009). Así, también, estudios con neuroimagen en pacientes con lesiones cerebrales sugieren la presencia de diferentes redes neuronales para cada componente de empatía (Cox et al., 2012). Para mayores alcances sobre los aspectos neuropsicológicos de la empatía puede revisarse a Arán Filippetti et al. (2012) y Shamay-Tsoory (2011). En síntesis, la empatía puede ser definida como un proceso vicario que incluye los componentes anteriores y que permite una mejor comprensión de los pensamientos y emociones de las personas (Davis, 2007; Jolliffe \& Farrington, 2004).

Si bien es en la infancia cuando aparecen las primeras conductas para detectar e imitar emociones de los demás, lo que sugiere una predisposición biológica de empatizar (Meltzoff \& Decety, 2003; Trevarthen \& Aitken, 2001), es la adolescencia también un periodo importante para el desarrollo de la empatía (Van der Graaff et al., 2014). En la adolescencia las oportunidades de establecer relaciones igualitarias dentro de la familia, la percepción de apoyo por parte de esta (Eisenberg \& Fabes, 1998; Garber, Robinson \& Valentiner, 1997) y el incremento de las interacciones con sus compañeros (Selman, 1981) permiten el establecimiento de un clima emocional adecuado que fomente el desarrollo de la empatía. Asimismo, la maduración cognitiva, la regulación de las emociones y las variaciones en la motivación ocurridas en la pubertad se encuentran relacionadas con el desarrollo de la empatía en la adolescencia (Zelazo, Craik \& Booth, 2004). Esta relación se logra a partir de la inhibición de la perspectiva personal que permite evaluar la perspectiva de las otras personas (Van der Graaff et al., 2014).

Estudios sobre empatía en adolescentes (Albiero, Matricardi, Speltri \& Toso, 2009) señalan su importancia en la adquisición y desarrollo de competencias sociales, favoreciendo un adecuado establecimiento y mantenimiento de amistades (del Barrio, Aluja \& García, 2004) y la calidad de las relaciones de cohesión y apoyo familiar (Guerney Jr., 1988; Henry, Sager \& Plunkett, 1996). Asimismo, se relaciona directamente con el desarrollo de la inteligencia social (Burke, 2001) y conductas prosociales en adolescentes (Eisenberg, Damon \& Lerner, 2006), convirtiéndose en agente protector de diversas formas de agresión (Jolliffe \& Farrington, 2004; Lovett \& Sheffield, 2007). En este sentido, en los adolescentes, bajos niveles o falta de empatía predicen la aparición de conductas de intimidación y acoso en sus diferentes formas, ya sea física, verbal, relacional, incluyendo el ciberbullying (Endresen \& Olweus, 2001; Gini, Albiero, Benelli \& Altoè, 2007; Jolliffe \& Farrington, 2006a), así como patrones característicos de personas acusadas por cometer violación sexual (Burke, 2001) y abuso infantil (Wiehe, 2003), entre otras conductas violentas.

$\mathrm{Al}$ considerar la importancia de la empatía para el desarrollo de adecuadas relaciones interpersonales y del bienestar en general de los adolescentes, resulta esencial contar con instrumentos que muestren adecuadas evidencias de validez y confiabilidad para su medición, con el objetivo de emplearlos tanto en investigación como en intervención (Albiero et al., 2009). En este sentido, en la actualidad los estudios emplean mayormente cuestionarios de autoinforme para la medición de la empatía (Albiero et al., 2009; Gerdes, Segal \& Lietz, 2010). Si bien las medidas de autoinformes pueden verse afectadas por sesgos subjetivos, es en la actualidad la estrategia más utilizada, debido a que implica una menor inversión económica, al mismo tiempo que brinda una amplia información (Choi \& Pak, 2005).

Entre los instrumentos para la medición de la empatía en adolescentes destaca la Basic Empathy Scale (BES por sus siglas en inglés; Jolliffe \& Farrington, 2006b). La BES evalúa la empatía desde una perspectiva cognitiva y emocional en adolescentes, teniendo como base la propuesta teórica de Cohen y Strayer (1996), quienes entienden la empatía como un proceso de comprensión de las emociones de otra persona. La BES supera las limitaciones metodológicas de otros instrumentos que evalúan parcialmente la empatía, como son 
la Escala de Empatía de Hogan (1969), que evalúa el componente cognitivo, o el Questionnaire Measure of Emotional Empathy (Mehrabian \& Epstein, 1972), que mide solamente la empatía emocional. Esta cualidad de la escala ha motivado diversos estudios acerca de su estructura factorial en diferentes países e idiomas, como China (Geng, Xia \& Qin, 2012), Italia (Albiero et al., 2009), Portugal (Pechorro, Ray, Salas-Wright, Maroco \& Gonçalves, 2015), Eslovaquia (Čavojová, Sirota \& Belovičová, 2012), Francia (Bensalah, Stefaniak, Carre \& Besche-Richard, 2016; D'Ambrosio, Olivier, Didon \& Besche, 2009), Perú (Merino-Soto \& GrimaldoMuchotrigo, 2015) y España (Oliva Delgado et al., 2011), que han confirmado la estructura bidimensional de la escala.

Todos los estudios anteriores reportaron haber eliminado una cantidad variable de ítems con el objetivo de obtener un modelo adaptable a la cultura, idioma y nivel lingüístico de la muestra de estudio (Villadangos, Errasti, Amigo, Jolliffe \& García-Cueto, 2016). Este es el caso del estudio realizado en España por Oliva Delgado et al. (2011), que tuvo como resultado una versión breve de la BES conformada por nueve ítems, denominada Basic Empathy Scale abreviada (BES abreviada). En la actualidad, la pertinencia de escalas más cortas se debe, en parte, a la presencia de periodos de atención más reducidos y el interés por obtener información de una gran cantidad de personas en menos tiempo y a través de medios como teléfonos móviles o redes sociales (Alam, Khusro, Rauf \& Zaman 2014; Konrath, Meier \& Bushman, 2018; Postmes, Haslam \& Jans, 2013). Desde una perspectiva psicométrica, la literatura sugiere que las escalas breves pueden ser tan válidas y confiables como los instrumentos que tienen mayor cantidad de ítems (Burisch, 1984; Loo \& Kelts, 1998).

La BES abreviada fue utilizada en el presente estudio y permite obtener una interpretación para las dimensiones cognitiva y afectiva. A nivel latinoamericano, solo un estudio ha trabajado con la BES abreviada (Merino-Soto \& Grimaldo-Muchotrigo, 2015), el cual señala que el mejor modelo explicativo del constructo empatía es el oblicuo de dos factores, que presenta indicadores de confiabilidad adecuados. Este estudio (Merino-Soto \& Grimaldo-Muchotrigo, 2015) concluye que no se justifica una interpretación a partir de un solo puntaje general, como otros autores señalaban.

Ahora bien, la literatura reporta consistentemente mayores niveles de empatía en mujeres, en comparación a los hombres, especialmente en el caso de la empatía afectiva (Albiero et al., 2009; Garaigordobil, 2009; Garaigordobil \& García de Galdeano, 2006; Jolliffe \& Farrington, 2006b; Lozano \& Etxebarria, 2007; Mestre Escrivá, Frías Navarro \& Samper García, 2004; Sánchez-Queija, Oliva \& Parra, 2006; Sánchez-Pérez, Fuentes, Jolliffe \& González-Salinas, 2014). Se sugiere que estas diferencias sexuales en la empatía surgen tempranamente alrededor de los 6 a 9 años de edad (Chapman et al., 2006) y que esta disparidad se amplía entre la primera infancia y la edad adulta (Michalska, Kinzler \& Decety, 2013). Las diferencias de la empatía en función al sexo pueden explicarse debido a los diferentes patrones de crianza de hombres y mujeres (Garaigordobil, 2009). En este sentido, se espera que los hombres respondan menos emocionalmente, mientras que las mujeres deberían tener habilidades para comprender y compartir los sentimientos y las emociones de otras personas (Lennon \& Eisenberg, 1987). Otros estudios señalan que la diferencia de la empatía entre hombres y mujeres ha sido evidente, tanto en la dimensión cognitiva y afectiva, es decir, las mujeres obtienen mayores niveles de empatía cognitiva y afectiva que los hombres (Lawrence, Shaw, Baker, Baron-Cohen \& David, 2004; Muncer \& Ling, 2006).

Dada la evidencia acerca de las diferencias sexuales en la empatía, es pertinente preguntarse si el sexo puede llevar a comprender de manera diferente los ítems de la BES abreviada y modificar la estructura teórica de la escala. Esto es importante, más aun sabiendo la falta de consistencia en las puntuaciones de empatía según sexo (Van der Graaff et al., 2014). En este sentido, el objetivo de este estudio fue analizar si la BES abreviada brinda interpretaciones válidas y es invariante entre ambos sexos.

El análisis de invarianza factorial es un procedimiento importante dentro de estudios que buscan realizar comparaciones entre dos o más grupos (e.g., sexo, edad, estado civil) haciendo uso de instrumentos de evaluación, permitiendo tener certeza de que las propiedades estructurales del instrumento se mantienen invariantes entre los grupos comparados (Byrne, 2008; van de Schoot, Lugtig \& Hox, 2012). Un elevado grado de invarianza en cuanto a la medición de un constructo permitiría concluir que la existencia o ausencia de diferencias observadas sería producto de la presencia o ausencia real de diferencias en el constructo evaluado, en este caso, la empatía (Caycho, 2017); por otro lado, si el grado de invarianza no es satisfactorio, es probable la presencia de sesgo de medida hacia uno de los grupos, probablemente debido a la presencia de factores desconocidos e irrelevantes (Pedraza \& Mungas, 2008), afectando la validez de las conclusiones derivadas de las comparaciones (Byrne, 2008). Así, la realización de estudios de comparación de un constructo solo es 
posible si existe evidencia empírica acerca de la equivalencia factorial entre los grupos (Elosua, 2005; Taylor, 2013; van de Schoot et al., 2012).

\section{Método}

\section{Diseño}

El estudio obedece a un diseño instrumental, debido a que se pretendió revisar las propiedades psicométricas de un instrumento de medida autoinformado (Montero \& León, 2005).

\section{Participantes}

La muestra del estudio fue de 934 estudiantes del nivel secundario de tres instituciones educativas de Lima Metropolitana, elegidos por conveniencia, debido a la accesibilidad a la muestra de estudio, de nivel sociocultural y económico medio. Se consideraron dos grupos de participantes: varones, que estuvieron integrados por 480 estudiantes con edades comprendidas entre los 11 y 18 años $(M=15,06$ años; $D E=1,57)$, y mujeres integradas por 454 estudiantes con edades comprendidas entre los 11 y 18 años $(M=14,94$ años; $D E=1,44)$. Se procuró que el tamaño de las muestras fuera similar, el cual es un requisito importante en estudio de invarianza factorial (Bollen, 1989).

\section{Instrumento}

Escala de Empatía Básica - Abreviada (BES abreviada; Oliva Delgado et al., 2011). Se utilizó la versión validada en el contexto peruano (Merino-Soto \& Grimaldo-Muchotrigo, 2015), que consta de nueve ítems escalados en formato Likert de cinco puntos $(1=$ Totalmente en desacuerdo, $2=$ En desacuerdo, $3=N i$ de acuerdo ni en desacuerdo, $4=$ De acuerdo, $5=$ Totalmente de acuerdo). La BES abreviada evalúa dos dimensiones de la empatía: empatía afectiva (ítems 1, 2, 3 y 6; e.g., "Me pongo triste cuando veo a gente llorando") y empatía cognitiva (ítems 4, 5, 7, 8 y 9; e.g., "Cuando alguien está deprimido suelo comprender cómo se siente"). Todos los ítems se califican de forma directa, por lo que una mayor puntuación refleja una mayor presencia del constructo evaluado. El estudio de validación peruano (Merino-Soto \& GrimaldoMuchotrigo, 2015) evaluó la estructura de la BES abreviada mediante la metodología de ecuaciones estructurales, presentando adecuados índices de bondad de ajuste, índice de $\chi^{2}=26,789$, raíz del residuo cuadrático medio estandarizado $(\mathrm{SRMR})=0,06$, raíz del error cuadrático medio de aproximación $(\mathrm{RMSEA})=0,015$, $90 \%$ IC $[0,0,0,07]$ e índice comparativo de Bentler-Bonett $(\mathrm{CFI})=0,99)$, y una consistencia interna aceptable, medida por omega de McDonald, para el factor empatía cognitiva $(\omega=0,81)$ y afectiva $(\omega=0,76)$.

\section{Procedimiento}

El protocolo del estudio y el instrumento de recolección de datos fueron aprobados por el Comité de Ética de la Universidad Privada del Norte. En primer lugar, se solicitaron los permisos respectivos a las autoridades de las instituciones educativas seleccionadas para la aplicación del instrumento, informándoles acerca del objetivo del estudio y las características de la BES abreviada. Una vez obtenida la autorización de las autoridades escolares, los estudiantes fueron informados acerca del objetivo de la investigación, obteniendo su participación voluntaria y asentimiento informado. De igual forma, se obtuvo el consentimiento firmado por sus padres para participar en el estudio.

La escala fue administrada de manera colectiva en las aulas de clases en un tiempo aproximado de 15 minutos por un grupo de estudiantes de los últimos años de la carrera de psicología de una universidad privada de Lima, quienes pasaron por un periodo de capacitación previo al trabajo de campo. Estos evaluadores permanecieron durante todo el proceso de aplicación del instrumento, brindando información necesaria o aclarando dudas de los estudiantes, sin influir en sus respuestas y verificando que los datos de identificación se llenaran satisfactoriamente. Al término de la evaluación, se agradeció a los estudiantes su participación.

\section{Análisis de Datos}

El análisis estadístico fue realizado mediante etapas. En la primera etapa se realizó el análisis descriptivo de los ítems y de sus características distribucionales. En la segunda etapa se evaluó la invarianza de la BES abreviada según el sexo (hombres y mujeres), para lo cual se utilizó un modelo de ecuaciones estructurales 
(SEM por sus siglas en inglés) en base al análisis factorial multigrupo con la librería "lavaan" (Rosseel et al., 2018). El método de estimación empleado fue los mínimos cuadrados ponderados robustos con media y varianza ajustada (WLSMV), debido a la naturaleza categórica de las variables en estudio (Brown, 2015), además de ser un estimador más confiable con muestras pequeñas, detectando relaciones estructurales con precisión en leve o moderada asimetría (Li, 2014). La estructura bidimensional fue establecida en base a un estudio previo (Merino-Soto \& Grimaldo-Muchotrigo, 2015), revisándose las bondades de ajuste de forma general y de acuerdo al género. Finalmente, se revisó la invarianza de medida. Para tal fin, se siguieron las recomendaciones expuestas por Byrne (2008), evaluándose progresivamente: la invarianza configuracional, que indica la estructura factorial sin consignar restricciones; la invarianza métrica, en sus tres niveles (Barrera-Barrera, Navarro-García \& Peris-Ortiz, 2015), métrica (cargas factoriales), fuerte (cargas factoriales e interceptos) y estricta (invarianza de cargas factoriales, interceptos y residuos). Además, fueron comparadas las medias latentes, debido a que se cumple la invarianza fuerte (Dimitrov, 2010).

Respecto a la evaluación de la invariancia configuracional, se utilizaron, de acuerdo a los criterios recomendados por Mueller y Hancock (2008), el $\chi^{2}$ y los índices absolutos RMSEA y SRMR, cuyos valores $\leq 0,06$ son considerados óptimos. También se usó CFI, para el cual valores por encima de 0,95 son aceptables (Hu \& Bentler, 1999).

Para evaluar la invarianza métrica, fuerte y estricta se tomaron en cuenta los cambios en el $\chi^{2}$. No obstante, como este depende del tamaño muestral (Fernández \& Díaz, 2000), también se consideró que variaciones del CFI $\left(\Delta_{\text {CFI }}\right) \leq 0,01$, RMSEA $\left(\Delta_{\text {RMSEA }}\right) \leq 0,015$, SRMR $\left(\Delta_{\text {SRMR }}\right) \leq 0,030$ son adecuados para aceptar la invarianza (Chen, 2007). De comprobarse la invarianza fuerte, se procedería a comparar las medias latentes (Dimitrov, 2010), por medio de $t$ de Student, y revisar los tamaños del efecto por medio de $d$ de Cohen (1992), siendo pequeño $d=0,20$, mediano $d=0,50$ y grande $d=0,80$.

Adicionalmente, se estimó la consistencia interna mediante el cálculo del coeficiente $\omega$ (McDonald, 1999), en vista de que es poco probable el cumplimiento del supuesto de tau-equivalencia para el uso del coeficiente alfa (Dominguez-Lara, 2016a).

\section{Resultados}

\section{Análisis Preliminar de los Ítems}

En primer lugar, en la Tabla 1 se reportan los estadísticos descriptivos de los ítems, evidenciando que los ítems $8(M=3,93)$ y $9(M=4,01)$ ostentan las medias aritméticas más altas, mientras que la media más baja está presente en el ítem $2(M=2,92)$. Respecto de la variabilidad, los ítems $3(D E=1,14)$ y $6(D E=1,25)$ muestran mayor dispersión. La asimetría y curtosis fluctuaron entre valores inferiores a $\pm 1,5$ (Pérez \& Medrano, 2010), indicando que la distribución de los ítems se aproxima a una distribución multivariada normal. Asimismo, la consistencia interna para el factor empatía cognitiva $(\omega=0,77)$ y afectiva $(\omega=0,79)$ es aceptable.

Tabla 1

Análisis Preliminar de los Ítems

\begin{tabular}{ccccccccc}
\hline \multirow{2}{*}{ Ítem } & \multicolumn{4}{c}{ Mujeres $(n=454)$} & \multicolumn{4}{c}{ Varones $(n=480)$} \\
\cline { 2 - 9 } & $M$ & $D E$ & $g_{1}$ & \multicolumn{1}{c}{$g_{2}$} & $M$ & $D E$ & \multicolumn{1}{c}{$g_{1}$} & \multicolumn{1}{c}{$g_{2}$} \\
\hline 1 & 3,39 & 1,02 & $-0,51$ & $-0,16$ & 2,91 & 1,09 & $-0,14$ & $-0,60$ \\
2 & 3,16 & 1,03 & $-0,12$ & $-0,39$ & 2,69 & 1,09 & 0,11 & $-0,70$ \\
3 & 3,50 & 1,03 & $-0,63$ & $-0,01$ & 2,99 & 1,18 & $-0,24$ & $-0,85$ \\
4 & 3,91 & 0,92 & $-0,81$ & 0,56 & 3,63 & 1,02 & $-0,72$ & 0,16 \\
5 & 3,58 & 0,96 & $-0,45$ & $-0,16$ & 3,33 & 1,08 & $-0,42$ & $-0,39$ \\
6 & 3,30 & 1,20 & $-0,29$ & $-0,76$ & 2,75 & 1,23 & 0,11 & $-0,95$ \\
7 & 3,54 & 0,98 & $-0,60$ & 0,03 & 3,33 & 0,97 & $-0,40$ & $-0,07$ \\
8 & 4,04 & 0,87 & $-0,99$ & 1,18 & 3,83 & 0,89 & $-0,94$ & 1,19 \\
9 & 4,12 & 0,83 & $-0,98$ & 1,18 & 3,91 & 0,91 & $-0,84$ & 0,64 \\
\hline
\end{tabular}

Nota. $M=$ Media; $D E=$ Desviación estándar; $g_{1}=$ Asimetría; $g_{2}=$ Curtosis. 


\section{Análisis de Invarianza}

Para la determinación de la invarianza de acuerdo al sexo, se evaluó progresivamente la invarianza configuracional $\left(M_{1}\right)$, invarianza métrica $\left(M_{2}\right)$, invarianza fuerte $\left(M_{3}\right)$ e invarianza estricta $\left(M_{4} ;\right.$ Byrne, 2008) con un estimador robusto (WLSMV), por la naturaleza categoría de las variables (Brown, 2015). De esa forma, se evaluó el ajuste del modelo base sin restricciones en ambos grupos por separado, obteniendo valores marginales (Tabla 2), pero similares entre varones y mujeres. Posteriormente, se analizó la estructura del BES entre los grupos $\left(M_{1}\right)$, teniendo como resultado excelentes valores en su conjunto, $\chi^{2}(52, N=934)=144,56$, $p<0,001, \mathrm{CFI}=0,98 \mathrm{SRMR}=0,04, \mathrm{RMSEA}=0,07,95 \%$ IC $[0,06,0,08]$. El $M_{1}$ es el modelo de referencia para la anidación de restricciones de los modelos $M_{2}, M_{3}$ y $M_{4}$.

Enseguida, fue analizada la invarianza métrica $\left(M_{2}\right)$, definida como el $M_{1}$ con restricciones sobre las cargas factoriales, hallándose índices de ajuste adecuados, CFI = 0,95, RMSEA = 0,08, 90\% IC [0,07, 0,09], $\mathrm{SRMR}=0,06$. Estos valores son similares (diferencia mínima entre índices de ajuste: $\triangle \mathrm{RMSEA}<0,015$, $\triangle \mathrm{CFI}<0,01$, SRMR < 0,030) a los valores del $M_{1}$ (Chen, 2007), indicando que no existen diferencias entre el modelo base $\left(M_{1}\right)$ y el modelo con la restricción de las cargas factoriales $\left(M_{2}\right)$, es decir, que las cargas factoriales son equivalentes (Tabla 2). Con esta condición, es posible comparar la invarianza de las covarianzas. Posteriormente, se evaluó la equivalencia entre interceptos (invarianza fuerte, $M_{3}$ ), en cuyo caso los índices reportaron un ajuste similar al modelo anterior, $\mathrm{CFI}=0,95, \mathrm{SRMR}=0,06, \mathrm{RMSEA}=0,06$, $90 \%$ IC $[0,05,0,07]$. Se observa que la diferencia, al comparar estos valores con los obtenidos en el modelo base $\left(M_{1}\right)$, son mínimas $(\Delta \leq 0,01$; Chen, 2007), aceptando la hipótesis de que los interceptos son invariantes. Finalmente, se analizó la invarianza estricta $\left(M_{4}\right)$, en la que se añade la invarianza de los residuos a la invarianza de cargas factoriales e interceptos, para la que los índices demuestran su invarianza.

Tabla 2

Invarianza de Medición de la Escala de Empatía

\begin{tabular}{|c|c|c|c|c|c|c|c|c|c|}
\hline Modelo & $\chi^{2}(g l)$ & $\begin{array}{c}\Delta \chi^{2} \\
(\Delta g l)\end{array}$ & $\begin{array}{l}\text { RMSEA } \\
{[90 \% \text { IC] }}\end{array}$ & $p$ & SRMR & CFI & $\Delta \mathrm{CFI}$ & $\triangle \mathrm{RMSEA}$ & $\Delta \mathrm{SRMR}$ \\
\hline Todo el grupo & $\begin{array}{c}144,56 \\
(26)\end{array}$ & - & $\begin{array}{c}0,07 \\
{[0,06,0,08]}\end{array}$ & $<0,001$ & 0,04 & 0,98 & - & - & - \\
\hline Mujeres & $\begin{array}{l}68,06 \\
(26)\end{array}$ & - & $\begin{array}{c}0,06 \\
{[0,04,0,08]}\end{array}$ & $<0,001$ & 0,04 & 0,97 & - & - & - \\
\hline Hombres & $\begin{array}{c}136,68 \\
(26)\end{array}$ & - & $\begin{array}{c}0,09 \\
{[0,08,0,11]}\end{array}$ & $<0,001$ & 0,06 & 0,94 & - & - & - \\
\hline$M_{1}$ & $\begin{array}{c}163,88 \\
(52)\end{array}$ & - & $\begin{array}{c}0,08 \\
{[0,07,0,09]}\end{array}$ & - & 0,06 & 0,95 & - & - & - \\
\hline$M_{2}$ & $\begin{array}{c}172,67 \\
(59)\end{array}$ & $\begin{array}{l}7,74 \\
(7)\end{array}$ & $\begin{array}{c}0,07 \\
{[0,06,0,09]}\end{array}$ & 0,357 & 0,06 & 0,95 & 0,00 & 0,00 & 0,00 \\
\hline$M_{3}$ & $\begin{array}{c}187,56 \\
(84)\end{array}$ & $\begin{array}{c}20,51 \\
(7)\end{array}$ & $\begin{array}{c}0,06 \\
{[0,05,0,07]}\end{array}$ & 0,719 & 0,06 & 0,95 & 0,00 & 0,01 & 0,00 \\
\hline$M_{4}$ & $\begin{array}{c}193,86 \\
(93)\end{array}$ & $\begin{array}{l}7,90 \\
(9)\end{array}$ & $\begin{array}{c}0,06 \\
{[0,05,0,07]}\end{array}$ & 0,540 & 0,06 & 0,95 & 0,00 & 0,00 & 0,00 \\
\hline
\end{tabular}

Nota. $M_{1}$ : Configuracional; $M_{2}$ : Métrica; $M_{3}$ : Fuerte; $M_{4}$ : Estricta. $N=934$

\section{Comparación de Medias Latentes en Función del Sexo}

Una vez comprobada la invarianza medida, se procedió a comparar las medias latentes, en vista al cumplimiento de la invarianza fuerte (Dimitrov, 2010). Por esa razón, se fijó la media del primer grupo en cero y la del segundo se dejó libre al parámetro para la diferencia. Los resultados revelan que existen diferencias tanto en la dimensión afectiva, $t(930,5)=9,25,0,001,95 \%$ IC $[0,37,0,58]$, como cognitiva, $t(932)=5,51, p<0,001,95 \%$ IC [0,14, 0,29], de acuerdo al sexo. Respecto a la primera dimensión, la media latente de las mujeres (media $=3,14, D E=0,34$ ) es superior a la de los varones (media $=2,66, D E=0,81$ ) y su diferencia puede ser considerada moderada $(d=0,60)$. Del mismo modo, en la dimensión cognitiva la media 
de mujeres (media $=3,54, D E=0,57$ ) es superior a la de varones (media $=3,33, D E=0,61$ ), aunque la magnitud de su diferencia es considerada pequeña $(d=0,36)$.

\section{Discusión}

La literatura no reporta estudios con la BES abreviada que comparen varones y mujeres desde un enfoque de invarianza. Este análisis es importante, más aun considerando que las características psicológicas de hombres y mujeres puede afectar de manera diferencial el comportamiento dependiendo del contexto (Hyde, 2005). Es así que el objetivo principal del estudio fue evaluar la invarianza factorial de la BES abreviada entre adolescentes peruanos en función al sexo. Siguiendo las recomendaciones de Abalo Piñeiro, Lévy Mangin, Rial Boubeta y Varela Mallou (2006) acerca de la estimación del mismo modelo en las muestras analizadas, y empleando la metodología SEM, en base al análisis factorial multigrupo, se revela que la estructura factorial de la escala es equivalente entre ambas muestras, confirmando, además, la invarianza en las cargas factoriales e interceptos. Este resultado muestra las primeras evidencias acerca de la ausencia de sesgo de medida de la BES abreviada, siendo igual de precisa tanto para varones como mujeres (Dimitrov, 2010). Si bien se reconoce que $M_{3}$ está al límite respecto de la diferencia mínima requerida $(\Delta \leq 0,01)$, estos puntos de corte se establecieron a partir del método de estimación de máxima verosimilitud y aquí se estimó con un método diagonalizado para variables ordinales WLSMV. Esta situación lleva a repensar los puntos de corte señalados por Chen (2007), lo que ha sido considerado recientemente a nivel científico (Xia, 2016).

El estudio confirmó, a través de la invarianza configuracional, que la estructura bidimensional en ambas submuestras resulta congruente con estudios previos (Merino-Soto \& Grimaldo-Muchotrigo, 2015; Oliva Delgado et al., 2011), ostentando valores de ajuste aceptables, parecidos a los de la versión original en diferentes países (Albiero et al., 2009; Bensalah et al., 2016; Čavojová et al., 2012; D'Ambrosio et al., 2009; Geng et al., 2012; Pechorro et al., 2015; Salas-Wright, Olate \& Vaughn, 2013). Asimismo, se acepta el modelo de invarianza métrica que permite concluir que las cargas factoriales son equivalentes entre ambos sexos. En un tercer momento, y aceptada la invarianza fuerte, los modelos (en hombres y mujeres) son equivalentes en sus cargas factoriales, interceptos y residuos.

De esta manera, la evidencia de que el constructo empatía tiende a comportarse de manera similar permite analizar modelos no ajustados a las características de hombres y mujeres. Así, los resultados llevan a señalar que el modelo de dos factores de empatía cuenta con atributos que lo hacen sólido y robusto a las diferencias existentes entre varones y mujeres, demostrando que, a pesar de los mayores niveles de empatía que las mujeres demuestran en comparación que los varones (Albiero et al., 2009; Garaigordobil, 2009; Garaigordobil \& García de Galdeano, 2006; Jolliffe \& Farrington, 2006a; Lozano \& Etxebarria, 2007; Mestre Escrivá et al., 2004; Sánchez-Queija et al., 2006; Sánchez-Pérez et al., 2014), el constructo es comprendido de igual manera por ambos grupos (Dominguez-Lara, 2016b). Estos hallazgos sugieren que las diferencias en las medias de los grupos son atribuibles a la empatía y no producto de variaciones en el instrumento de medida (Hirschfeld \& Von Brachel, 2014). De esta manera, se justifica el empleo de la BES abreviada en estudios comparativos por sexo, con el objetivo de estudiar sus efectos sobre variables relacionadas con el bienestar.

Los resultados muestran diferencias entre hombres y mujeres, ya que las dimensiones afectiva y cognitiva de la empatía se encuentran a favor de las mujeres. Esto está relacionado con lo señalado por la literatura previa (Lawrence et al., 2004; Mestre, Samper, Frías \& Tur, 2009; Muncer \& Ling, 2006). Así, aunque tanto hombres como mujeres comprenden igualmente la empatía, pareciera que las mujeres tienden a expresarse más afectivamente (Retuerto Pastor, 2004). Según algunos autores (Batson, Fultz \& Schoenrade, 1987/1992), estos resultados son explicados por la percepción relacionada con una serie de estereotipos sociales de que la empatía es una característica más frecuente en mujeres en comparación a los hombres, debido a su mayor sensibilidad, capacidad de apoyo y detección de sentimientos, entre otras. Esto hace suponer que las mujeres, en mayor medida que los hombres, han sido socializados para favorecer la expresión de habilidades orientadas hacia la calidez en las relaciones interpersonales (Garaigordobil, 2009). Desde una perspectiva neuropsicológica, otros autores (Han, Fan \& Mao, 2008) señalan que estas diferencias podrían ser el resultado de cómo hombres y mujeres muestran diferentes patrones en la activación de redes cerebrales en dos situaciones: primero, al momento de evaluar su propia respuesta emocional ante la emoción expresada en el rostro de otra persona y, segundo, cuando evalúan el estado emocional expresado por otras personas.

Estos resultados no concuerdan con estudios que sugieren que hombres y mujeres están igualmente propensos a comprender los sentimientos de los demás antes de actuar (Broidy, Cauffman, Espelage, Mazerolle \& Piquero, 2003). Además, se reporta que la magnitud de las diferencias de la empatía entre 
mujeres y hombres parece ser diferente según el periodo de edad estudiado, con mayores diferencias en edades más avanzadas (Mestre et al., 2009), y en relación al método de medición (Eisenberg \& Lennon, 1983).

En este sentido, los hallazgos en este estudio y otros sugieren que el problema de las diferencias de sexo respecto de la empatía sigue sin resolverse. Esto hace necesario que los estudios sobre este tema deban considerar aún la hipótesis de similitud de la empatía en función del sexo de los adolescentes (Hyde, 2005). Comprender adecuadamente las diferencias de la empatía entre hombres y mujeres es importante para implementar programas centrados en el desarrollo de la misma (Mestre et al., 2009).

Los resultados deben examinarse a la luz de algunas limitaciones, por lo que no pueden considerarse definitivos. Primero, este estudio estuvo restringido a adolescentes de la ciudad de Lima, lo que afecta la generalización de los hallazgos. Se necesita que investigaciones futuras puedan ampliar la medición de la empatía en grupos de adolescentes de diferentes regiones del Perú. Esto lleva a considerar también el estudio de la invarianza de medición de la BES abreviada en diferentes regiones para garantizar la comparabilidad de su estructura. Se deben realizar estudios de replicación con el fin de conocer si el grado de invarianza encontrado obedece más a características de la muestra estudiada o al azar. Del mismo modo, no está claro que si el estudio se replicara en otros países hispanoparlantes los resultados pudieran diferir.

Segundo, la naturaleza transversal del estudio impidió examinar la estabilidad y la evolución de la estructura de dos factores de la BES abreviada en varones y mujeres a través del tiempo. Estudios futuros con un diseño longitudinal deberían abordar esta limitación y evaluar ciertas tendencias del desarrollo de la empatía en ambos sexos. Por otro lado, sería recomendable el contraste con criterios externos (p.e., otros instrumentos que evalúen empatía o constructos afines) para brindarle mayor fortaleza a las conclusiones derivadas de los resultados de la BES abreviada.

A pesar de las limitaciones, los hallazgos del estudio presentan importantes fortalezas. Primero, se proporciona información importante y sólida sobre la aplicabilidad sin sesgo de la BES abreviada, debido al número aproximadamente igual de ambos sexos. Segundo, en base a la revisión de la literatura, este es el primer estudio que ha examinado la invarianza factorial de la BES abreviada en función del sexo. Además, aunque solo se pudo demostrar invarianza factorial parcial, los resultados son una contribución importante a la medición de la empatía en la adolescencia.

En resumen, los resultados proporcionan evidencia parcial de que la BES abreviada presenta invarianza factorial para ser utilizada como medida de la empatía en adolescentes hombres y mujeres.

\section{Referencias}

Abalo Piñeiro, J., Lévy Mangin, J. P., Rial Boubeta, A. \& Varela Mallou, J. (2006). Invarianza factorial con muestras múltiples. En J. P. Lévy Mangin (Dir.) \& J. Varela Mallou (Coord.), Modelización con estructuras de covarianzas en ciencias sociales: temas esenciales, avanzados y aportaciones especiales (pp. 259-278). Madrid, España: Netbiblo.

Alam, I., Khusro, S., Rauf, A. \& Zaman, Q. (2014). Conducting surveys and data collection: From traditional to mobile and SMS-based surveys. Pakistan Journal of Statistics and Operation Research, 10, 169-187. https://doi.org/10.18187/pjsor.v10i2.758

Albiero, P., Matricardi, G., Speltri, D. \& Toso, D. (2009). The assessment of empathy in adolescence: A contribution to the Italian validation of the "Basic Empathy Scale". Journal of Adolescence, 32, 393-408. https://doi.org/10.1016/j.adolescence.2008.01.001

Arán Filippetti, V., López, M. B. \& Richaud, M. C. (2012). Aproximación neuropsicológica al constructo de empatía: aspectos cognitivos y neuroanatómicos. Cuadernos de Neuropsicología, 6(1), 63-83. https://doi.org/10.7714/cnps/6.1.204

Barrera-Barrera, R., Navarro-García, A. \& Peris-Ortiz, M. (2015). El papel de la invarianza factorial en la validación del constructo calidad de servicio electrónico. Revista Europea de Dirección y Economía de la Empresa, 24, 13-24. https://doi.org/10.1016/j.redee.2014.07.001

Batson, C. D., Fultz, J. \& Schoenrade, P. A. (1987/1992). Las reacciones emocionales de los adultos ante el malestar ajeno (I. Aizpurúa, Trad.; Título original: Adults' emotional reactions to the distress of others). En N. Eisenberg \& J. Strayer (Eds.), La empatía y su desarrollo (I. Aizpurúa, Trad.; Título original: Empathy and its development; pp. 181-204). Bilbao, España: Desclée de Brouwer.

Bensalah, L., Stefaniak, N., Carre, A. \& Besche-Richard, C. (2016). The Basic Empathy Scale adapted to French middle childhood: Structure and development of empathy. Behavior Research Methods, 48, 1410-1420. https://doi.org/10.3758/s13428-015-0650-8

Bollen, K. A. (1989). Structural equations with latent variables. New York, NY: John Wiley \& Sons.

Borrell i Carrió, F. (2004). Entrevista clínica: manual de estrategias prácticas. Barcelona, España: Sociedad Española de Medicina de Familia y Comunitaria.

Broidy, L., Cauffman, E., Espelage, D. L., Mazerolle, P. \& Piquero, A. (2003). Sex differences in empathy and its relation to juvenile offending. Violence and Victims, 18, 503-516. https://doi.org/10.1891/vivi.2003.18.5.503

Brown, T. A. (2015). Confirmatory factor analysis for applied research (2a ed.). New York, NY: Guilford Press.

Burisch, M. (1984). You don't always get what you pay for: Measuring depression with short and simple versus long and sophisticated scales. Journal of Research in Personality, 18, 81-98. https://doi.org/10.1016/0092-6566(84)90040-0

Burke, D. M. (2001). Empathy in sexually offending and nonoffending adolescent males. Journal of Interpersonal Violence, 16, $222-233$. https://doi.org/10.1177/088626001016003003

Byrne, B. M. (2008). Testing for multigroup equivalence of a measuring instrument: A walk through the process. Psicothema, 20, 872882. Extraído de http://www.psicothema.com/PDF/3569.pdf 
Campbell, R. G. \& Babrow, A. S. (2004). The role of empathy in responses to persuasive risk communication: Overcoming resistance to HIV prevention messages. Health Communication, 16, 159-182. https://doi.org/10.1207/S15327027HC1602_2

Čavojová, V., Sirota, M. \& Belovičová, Z. (2012). Slovak validation of the Basic Empathy Scale in pre-adolescents. Studia Psychologica, 54, 195-208. Extraído de http://www.studiapsychologica.com/uploads/CAVOJOVA_SP_3_vol.54_2012_pp.195-208.pdf

Caycho, T. (2017). Importancia del análisis de invarianza factorial en estudios comparativos en ciencias de la salud. Educación Médica Superior, 31(2), 1-3. Extraído de http://scielo.sld.cu/pdf/ems/v31n2/ems04217.pdf

Chapman, E., Baron-Cohen, S., Auyeung, B., Knickmeyer, R., Taylor, K. \& Hackett, G. (2006). Fetal testosterone and empathy: Evidence from the empathy quotient (EQ) and the "Reading the Mind in the Eyes" test. Social Neuroscience, 1, 135-148. https://doi.org/10.1080/17470910600992239

Chen, F. F. (2007). Sensitivity of goodness of fit indexes to lack of measurement invariance. Structural Equation Modeling, 14, 464-504. https://doi.org/10.1080/10705510701301834

Choi, B. C. K. \& Pak, A. W. P. (2005). A catalog of biases in questionnaires. Preventing Chronic Disease, 2(1), Artículo 13. Extraído de https://www.ncbi.nlm.nih.gov/pmc/articles/PMC1323316/

Cohen, D. \& Strayer, J. (1996). Empathy in conduct-disordered and comparison youth. Developmental Psychology, 32, 988-998. https://doi.org/10.1037/0012-1649.32.6.988

Cohen, J. (1992). A power primer. Psychological Bulletin, 112, 155-159. https://doi.org/10.1037/0033-2909.112.1.155

Cox, C. L., Uddin, L. Q., Di Martino, A., Castellanos, F. X., Milham, M. P. \& Kelly, C. (2012). The balance between feeling and knowing: Affective and cognitive empathy are reflected in the brain's intrinsic functional dynamics. Social Cognitive and Affective Neuroscience, 7, 727-737. https://doi.org/10.1093/scan/nsr051

D'Ambrosio, F., Olivier, M., Didon, D. \& Besche, C. (2009). The Basic Empathy Scale: A French validation of a measure of empathy in youth. Personality and Individual Differences, 46, 160-165. https://doi.org/10.1016/j.paid.2008.09.020

Davis, M. H. (2007). Empathy. En J. E. Stets \& J. H. Turner (Eds.), Handbook of the sociology of emotions (pp. 443-466). New York, NY: Springer.

Decety, J. (2010). The neurodevelopment of empathy in humans. Developmental Neuroscience, 32, 257-267. https://doi.org/10.1159/000317771

Decety, J. \& Jackson, P. L. (2004). The functional architecture of human empathy. Behavioral and Cognitive Neuroscience Reviews, 3, 71-100. https://doi.org/10.1177/1534582304267187

del Barrio, V., Aluja, A. \& García, L. F. (2004). Relationship between empathy and the big five personality traits in a sample of Spanish adolescents. Social Behavior and Personality, 32, 677-681. https://doi.org/10.2224/sbp.2004.32.7.677

Dimberg, U. \& Thunberg, M. (2012). Empathy, emotional contagion, and rapid facial reactions to angry and happy facial expressions. PsyCh Journal, 1, 118-127. https://doi.org/10.1002/pchj.4

Dimberg, U., Thunberg, M. \& Elmehed, K. (2000). Unconscious facial reactions to emotional facial expressions. Psychological Science, 11, 86-89. https://doi.org/10.1111/1467-9280.00221

Dimitrov, D. M. (2010). Testing for factorial invariance in the context of construct validation. Measurement and Evaluation in Counseling and Development, 43, 121-149. https://doi.org/10.1177/0748175610373459

Dominguez-Lara, S. A. (2016a). Secretos del coeficiente alfa. Actas Urológicas Españolas, 40, 471. https://doi.org/10.1016/j.acuro.2016.04.002

Dominguez-Lara S. A. (2016b). Comparación del autoconcepto entre grupos, ¿sesgo o diferencias?: comentarios a Castillo et al. Revista Chilena de Pediatría, 87, 436. https://doi.org/10.1016/j.rchipe.2016.03.003

Eisenberg, N., Damon, W. \& Lerner, R. M. (2006). Handbook of child psychology: Social, emotional, and personality development (6a ed.). New York, NY: John Wiley \& Sons.

Eisenberg, N. \& Fabes, R. A. (1998). Prosocial development. En W. Damon \& N. Eisenberg (Eds.), Handbook of child psychology, Vol. 3: Social, emotional, and personality development (5a ed., pp. 701-778). New York, NY: John Wiley \& Sons.

Eisenberg, N. \& Lennon, R. (1983). Sex differences in empathy and related capacities. Psychological Bulletin, 94, $100-131$. https://doi.org/10.1037/0033-2909.94.1.100

Eisenberg, N., Shea, C. L., Carlo, G. \& Knight, G. P. (1991). Empathy-related responding and cognition: A "chicken and the egg" dilemma. En W. M. Kurtines \& J. L. Gewirtz (Eds.), Handbook of moral behavior and development. Volume 2: Research (pp. 63-88). Hillsdale, NJ: Lawrence Erlbaum.

Elosua, P. (2005). Evaluación progresiva de la invarianza factorial entre las versiones original y adaptada de una escala de autoconcepto. Psicothema, 17, 356-362. Extraído de https://www.redalyc.org/pdf/727/72717227.pdf

Endresen, I. M. \& Olweus, D. (2001). Self-reported empathy in Norwegian adolescents: Sex differences, age trends, and relationship to bullying. En A. C. Bohart \& D. J. Stipek (Eds.), Constructive \& destructive behavior: Implications for family, school, \& society (pp. 147-165). Washington, DC: American Psychological Association. https://doi.org/10.1037/10433-007

Fernández, P. \& Díaz, P. (2000). Significancia estadística y relevancia clínica. Cadena de Atención Primaria, 8, 191-195. Extraído de https://www.fisterra.com/mbe/investiga/signi_estadi/signi_estadisti2.pdf

Fernández-Pinto, I., López-Pérez, B. \& Márquez, M. (2008). Empatía: medidas, teorías y aplicaciones en revisión. Anales de Psicología, 24, 284-298. Extraído de https://www.redalyc.org/pdf/167/16711589012.pdf

Garaigordobil, M. (2009). A comparative analysis of empathy in childhood and adolescence: Gender differences and associated socioemotional variables. International Journal of Psychology and Psychological Therapy, 9, 217-235. Extraído de https://www.ijpsy.com/volumen9/num2/233/a-comparative-analysis-of-empathy-in-childhood-EN.pdf

Garaigordobil, M. \& García de Galdeano, P. (2006). Empatía en niños de 10 a 12 años. Psicothema, 18, 180-186. Extraido de http://www.psicothema.es/pdf/3195.pdf

Garber, J., Robinson, N. S. \& Valentiner, D. (1997). The relation between parenting and adolescent depression: Self-worth as a mediator. Journal of Adolescent Research, 12, 12-33. https://doi.org/10.1177/0743554897121003

Geng, Y., Xia, D. \& Qin, B. (2012). The Basic Empathy Scale: A Chinese validation of a measure of empathy in adolescents. Child Psychiatry \& Human Development, 43, 499-510. https://doi.org/10.1007/s10578-011-0278-6

Gerdes, K. E., Segal, E. A. \& Lietz, C. A. (2010). Conceptualising and measuring empathy. The British Journal of Social Work, 40, 2326-2343. https://doi.org/10.1093/bjsw/bcq048

Gini, G., Albiero, P., Benelli, B. \& Altoè, G. (2007). Does empathy predict adolescents' bullying and defending behavior? Aggressive Behavior, 33, 467-476. https://doi.org/10.1002/ab.20204

Guerney Jr., B. G. (1988). Family relationship enhancement: A skill training approach. En L. A. Bond \& B. M. Wagner (Eds.), Families in transition: Primary prevention programs that work, Vol. 11 (pp. 99-134). Beverly Hills, CA: SAGE. 
Guzmán González, M., Péloquin, K., Lafontaine, M. -F., Trabucco, C. \& Urzúa, A. (2014). Evaluación de la empatía diádica: análisis de las propiedades psicométricas del Índice de Reactividad Interpersonal en Parejas (IRIC-C) en contexto chileno. Psicoperspectivas, 13(2), 156-164. https://doi.org/10.5027/psicoperspectivas-Vol13-Issue2-fulltext-340

Han, S., Fan, Y. \& Mao, L. (2008). Gender difference in empathy for pain: An electrophysiological investigation. Brain Research, 1196, 85-93. https://doi.org/10.1016/j.brainres.2007.12.062

Hemmerdinger, J. M., Stoddart, S. D. R. \& Lilford, R. J. (2007). A systematic review of tests of empathy in medicine. BMC Medical Education, 7, Artículo 24. https://doi.org/10.1186/1472-6920-7-24

Henry, C. S., Sager, D. W. \& Plunkett, S. W. (1996). Adolescents' perceptions of family system characteristics, parent-adolescent dyadic behaviors, adolescent qualities, and adolescent empathy. Family Relations, 45, 283-292. https://doi.org/10.2307/585500

Hirschfeld, G. \& von Brachel, R. (2014). Multiple-group confirmatory factor analysis in R-A tutorial in measurement invariance with continuous and ordinal indicators. Practical Assessment, Research \& Evaluation, 19, Artículo 7. Extraído de https://pareonline.net/pdf/v19n7.pdf

Hogan, R. (1969). Development of an empathy scale. Journal of Counsulting and Clinical Psychology, 33, 307-316. https://doi.org/10.1037/h0027580

Hu, L. \& Bentler, P. M. (1999). Cutoff criteria for fit indexes in covariance structure analysis: Conventional criteria versus new alternatives. Structural Equation Modeling, 6, 1-55. https://doi.org/10.1080/10705519909540118

Hyde, J. S. (2005). The gender similarities hypothesis. American Psychologist, 60, 581-592. https://doi.org/10.1037/0003-066X.60.6.581

Jolliffe, D. \& Farrington, D. P. (2004). Empathy and offending: A systematic review and meta-analysis. Aggression and Violent Behavior, 9, 441-476. https://doi.org/10.1016/j.avb.2003.03.001

Jolliffe, D. \& Farrington, D. P. (2006a). Examining the relationship between low empathy and bullying. Aggressive Behavior, 32, 540550. https://doi.org/10.1002/ab.20154

Jolliffe, D. \& Farrington, D. P. (2006b). Development and validation of the Basic Empathy Scale. Journal of Adolescence, 29, 589-611. https://doi.org/10.1016/j.adolescence.2005.08.010

Jolliffe, D. \& Murray, J. (2012). Lack of empathy and offending: Implications for tomorrow's research and practice. En R. Loeber \& B. C. Welsh (Eds.), The future of criminology (pp. 62-69). New York, NY: Oxford University Press.

Konrath, S., Meier, B. P. \& Bushman, B. J. (2018). Development and validation of the single item trait empathy scale (SITES). Journal of Research in Personality, 73, 111-122. https://doi.org/10.1016/j.jrp.2017.11.009

Lawrence, E. J., Shaw, P., Baker, D., Baron-Cohen, S. \& David, A. S. (2004). Measuring empathy: Reliability and validity of the Empathy Quotient. Psychological Medicine, 34, 911-920. https://doi.org/10.1017/S0033291703001624

Lennon, R. \& Eisenberg, N. (1987). Gender and age differences in empathy and sympathy. En N. Eisenberg \& J. Strayer (Eds.), Empathy and its development (pp. 195-217). New York, NY: Cambridge University Press.

$\mathrm{Li}, \mathrm{C}$. -H. (2014). The performance of MLR, USLMV, and WLSMV estimation in structural regression models with ordinal variables (Disertación de Doctorado, Michigan State University, East Lansing, MI, Estados Unidos). Extraído de https://d.lib.msu.edu/islandora/object/etd:3268/datastream/OBJ/download/The_performance_of_MLR_USLMV_and_WLSMV_es timation_in_structural_regression_models_with_ordinal_variables.pdf

Loo, R. \& Kelts, P. (1998). A caveat on using single-item measures. Employee Assistance Quarterly, 14(2), 75-80. https://doi.org/10.1300/J022v14n02_06

Lovett, B. J. \& Sheffield, R. A. (2007). Affective empathy deficits in aggressive children and adolescents: A critical review. Clinical Psychology Review, 27, 1-13. https://doi.org/10.1016/j.cpr.2006.03.003

Lozano, A. M. \& Etxebarria, I. (2007). La tolerancia a la diversidad en los adolescentes y su relación con la autoestima, la empatía y el concepto del ser humano. Infancia y Aprendizaje, 30, 109-129. https://doi.org/10.1174/021037007779849673

McDonald, R. P. (1999). Test theory: A unified treatment. Mahwah, NJ: Lawrence Erlbaum.

Mehrabian, A. \& Epstein, N. (1972). A measure of emotional empathy1. Journal of Personality, 40, 525-543. https://doi.org/10.1111/j.14676494.1972.tb00078.x

Meltzoff, A. N. \& Decety, J. (2003). What imitation tells us about social cognition: A rapprochement between developmental psychology and cognitive neuroscience. Philosophical Transactions of the Royal Society B: Biological Sciences, 358, 491-500. https://doi.org/10.1098/rstb.2002.1261

Merino-Soto, C. \& Grimaldo-Muchotrigo, M. (2015). Validación estructural de la Escala Básica de Empatía (Basic Empathy Scale) modificada en adolescentes: un estudio preliminar. Revista Colombiana de Psicología, 24, 261-270. https://doi.org/10.15446/rcp.v24n2.42514

Mestre, M. V., Samper, P., Frías, M. D. \& Tur, A. M. (2009). Are women more empathetic than men? A longitudinal study in adolescence. The Spanish Journal of Psychology, 12, 76-83. https://doi.org/10.1017/S1138741600001499

Mestre Escrivá, V., Frías Navarro, M. D. \& Samper García, P. (2004). La medida de la empatía: análisis del Interpersonal Reactivity Index. Psicothema, 16, 255-260. Extraído de http://www.psicothema.com/pdf/1191.pdf

Michalska, K. J., Kinzler, K. D. \& Decety, J. (2013). Age-related sex differences in explicit measures of empathy do not predict brain responses across childhood and adolescence. Developmental Cognitive Neuroscience, 3, 22-32. https://doi.org/10.1016/j.dcn.2012.08.001

Montero, I. \& León, O. G. (2005). Sistema de clasificación del método en los informes de investigación en psicología. International Journal of Clinical and Health Psychology, 5, 115-127. Extraído de https://www.redalyc.org/html/337/33701007/

Mueller, R. O. \& Hancock, G. R. (2008). Best practices in structural equation modeling. En J. Osborne (Ed.), Best practices in quantitative methods (pp. 488-508). Thousand Oaks, CA: SAGE. https://doi.org/10.4135/9781412995627.d38

Muncer, S. J. \& Ling, J. (2006). Psychometric analysis of the Empathy Quotient (EQ) scale. Personality and Individual Differences, 40, 1111-1119. https://doi.org/10.1016/j.paid.2005.09.020

Neumann, M., Scheffer, C., Tauschel, D., Lutz, G., Wirtz, M. \& Edelhäuser, F. (2012). Physician empathy: Definition, outcome-relevance and its measurement in patient care and medical education. GMS Zeitschrift für Medizinische Ausbildung, 29 , Doc11. https://doi.org/10.3205/zma000781

Oliva Delgado, A., Antolín Suárez, L., Pertegal Vega, M. Á., Ríos Bermúdez, M., Parra Jiménez, Á., Hernando Gómez, Á. \& Reina Flores, M. C. (2011). Instrumentos para la evaluacion de la salud mental y el desarrollo positivo adolescente y los activos que lo promueven. Sevilla, España: Consejería de Salud.

Pechorro, P., Ray, J. V., Salas-Wright, C. P., Maroco, J. \& Gonçalves, R. A. (2015). Adaptation of the Basic Empathy Scale among a Portuguese sample of incarcerated juvenile offenders. Psychology, Crime \& Law, 21, 699-714. https://doi.org/10.1080/1068316X.2015.1028546

Pedersen, R. (2008). Empathy: A wolf in sheep's clothing? Medicine, Health Care and Philosophy, 11, 325-335. https://doi.org/10.1007/s11019-007-9104-0

Pedraza, O. \& Mungas, D. (2008). Measurement in cross-cultural neuropsychology. Neuropsychology Review, 18, $184-193$. https://doi.org/10.1007/s11065-008-9067-9 
Pérez, E. R. \& Medrano, L. (2010). Análisis factorial exploratorio: bases conceptuales y metodológicas. Revista Argentina de Ciencias del Comportamiento, 2(1), 58-66. Extraído de http://www.redalyc.org/pdf/3334/333427068006.pdf

Postmes, T., Haslam, S. A. \& Jans, L. (2013). A single-item measure of social identification: Reliability, validity, and utility. British Journal of Social Psychology, 52, 597-617. https://doi.org/10.1111/bjso.12006

Retuerto Pastor, Á. (2004). Diferencias en empatía en función de las variables género y edad. Apuntes de Psicología, 22, 323-339. Extraído de http://www.apuntesdepsicologia.es/index.php/revista/article/view/59/61

Rey, C. (2003). La medición de la empatía en preadolescentes y adolescentes varones: adaptación y validación de una escala. Revista Latinoamericana de Psicología, 35, 185-194. Extraído de https://www.redalyc.org/pdf/805/80535206.pdf

Rosseel, Y., Oberski, D., Byrnes, J., Vanbrabant, L., Savalei, V., Merkle, E. ... Jorgensen, T. D. (2018). Package 'lavaan' 0.6-3. Extraído de https://cran.r-project.org/web/packages/lavaan/lavaan.pdf

Salas-Wright, C. P., Olate, R. \& Vaughn, M. G. (2013). Assessing empathy in Salvadoran high-risk and gang-involved adolescents and young adults: A Spanish validation of the Basic Empathy Scale. International Journal of Offender Therapy and Comparative Criminology, 57, 1393-1416. https://doi.org/10.1177/0306624X12455170

Sánchez-Pérez, N., Fuentes, L. J., Jolliffe, D. \& González-Salinas, C. (2014). Assessing children's empathy through a Spanish adaptation of the Basic Empathy Scale: Parent's and child's report forms. Frontiers in Psychology, 5, Artículo 1438. https://doi.org/10.3389/fpsyg.2014.01438

Sánchez-Queija, I., Oliva, A. \& Parra, Á. (2006). Empatía y conducta prosocial durante la adolescencia. Revista de Psicología Social, 21, 259-271. https://doi.org/10.1174/021347406778538230

Selman, R. L. (1981). The development of interpersonal competence: The role of understanding in conduct. Developmental Review, 1, 401-422. https://doi.org/10.1016/0273-2297(81)90034-4

Shamay-Tsoory, S. G. (2011). The neural bases for empathy. The Neuroscientist, 17, 18-24. https://doi.org/10.1177/1073858410379268

Shamay-Tsoory, S. G., Aharon-Peretz, J. \& Perry, D. (2009). Two systems for empathy: A double dissociation between emotional and cognitive empathy in inferior frontal gyrus versus ventromedial prefrontal lesions. Brain, 132, 617-627. https://doi.org/10.1093/brain/awn279

Shirtcliff, E. A., Dahl, R. E. \& Pollak, S. D. (2009). Pubertal development: Correspondence between hormonal and physical development. Child Development, 80, 327-337. https://doi.org/10.1111/j.1467-8624.2009.01263.x

Sulzer, S. H., Feinstein, N. W. \& Wendland, C. L. (2016). Assessing empathy development in medical education: A systematic review. Medical Education, 50, 300-310. https://doi.org/10.1111/medu.12806

Taylor, C. S. (2013). Validity and validation. New York, NY: Oxford University Press.

Trevarthen, C. \& Aitken, K. J. (2001). Infant intersubjectivity: Research, theory, and clinical applications. Journal of Child Psychology and Psychiatry, 42, 3-48. https://doi.org/10.1111/1469-7610.00701

van Boxtel, A. (2010). Facial EMG as a tool for inferring affective states. En A. J. Spink, F. Grieco, O. E. Krips, L. W. S. Loijens, L. P. J. J. Noldus \& P. H. Zimmerman (Eds.), Proceedings of Measuring Behavior 2010, $7^{\text {th }}$ International Conference on Methods and Techniques in Behavioral Research (pp. 104-108). Wageningen, Holanda: Noldus Information Technology.

van de Schoot, R., Lugtig, P. \& Hox, J. (2012). A checklist for testing measurement invariance. European Journal of Developmental Psychology, 9, 486-492. https://doi.org/10.1080/17405629.2012.686740

Van der Graaff, J., Branje, S., De Wied, M., Hawk, S., Van Lier, P. \& Meeus, W. (2014). Perspective taking and empathic concern in adolescence: Gender differences in developmental changes. Developmental Psychology, 50, 881-888. https://doi.org/10.1037/a0034325

Villadangos, M., Errasti, J., Amigo, I., Jolliffe, D. \& García-Cueto, E. (2016). Characteristics of empathy in young people measured by the Spanish validation of the Basic Empathy Scale. Psicothema, 28, 323-329. Extraído de http://digibuo.uniovi.es/dspace/bitstream/10651/38566/1/Psicothema,2016,28(3),323-29.pdf

Wiehe, V. R. (2003). Empathy and narcissism in a sample of child abuse perpetrators and a comparison sample of foster parents. Child Abuse \& Neglect, 27, 541-555. https://doi.org/10.1016/S0145-2134(03)00034-6

Xia, Y. (2016). Investigating the chi-square-based model-fit indexes for WLSMV and ULSMV estimators. Tallahassee, FL: Florida State University. Extraído de http://purl.flvc.org/fsu/fd/FSU_2016SU_Xia_fsu_0071E_13379

Zelazo, P. D., Craik, F. I. M. \& Booth, L. (2004). Executive function across the life span. Acta Psychologica, 115, 167-183. https://doi.org/10.1016/j.actpsy.2003.12.005

Fecha de recepción: Mayo de 2018.

Fecha de aceptación: Octubre de 2018. 